\title{
Multi-constrained topology optimization using constant criterion surface algorithm
}

\author{
M. MRZYGŁÓD* \\ Institute of Rail Vehicles, Cracow University of Technology, 37 Jana Pawła II Ave., 31-864 Kraków, Poland
}

\begin{abstract}
This paper sets out to describe a multi-constrained approach to topology optimization of structures. In the optimization, a constant criterion surface algorithm and the multi-constraint procedure is used. The multi-constraint procedure consists of constraints normalization and equivalent design space assembling. The work is illustrated by an example of the L-shaped domain optimization with the horizontal line support and complex loads. The example takes into consideration stress, fatigue and compliance constraints. The separate and simultaneous application of constraints resulted in significant differences in structure topology layouts. The application of a fatigue constraint gave more conservative results when compared to static stress or compliance limitations. The multi-constrained approach allowed effectively lowering the mass of the structure while satisfying all constraints.
\end{abstract}

Key words: multi-constrained topology optimization, stress and fatigue constraints, constant criterion surface algorithm.

\section{Introduction}

The topology optimization nowadays can give an answer to the demand for solving large and multi-load problems. In popular methods like SIMP (solid isotropic material with penalization) [1] and ESO (evolutionary structural optimization) [2], the most often compliance and stress constraints are used [3]. Rarely, we can find more complex constraints like fatigue $[4,5]$ or reliability [6].The stress constraint usually is added to displacement-based optimization procedures by resorting to post-processing operations [7]. There are only a few propositions in the literature of multi-constrained topology optimization methods. An application of a weighted sum of normed constraints was proposed by Min et al. to generate structures satisfying static and vibration performance measures [8]. A similar weighted method was used by Lee et al. in multicriteria optimization of automotive bodies [9]. A single aggregated ranking parameter approach was proposed by Ramani [10]. He showed examples of topology optimization with multiple materials and a few constraints.

In this paper, investigation on the multi-constrained structure optimization with the usage of a constant criterion surface algorithm (CCSA) is presented.

\section{The constant criterion surface algorithm}

The optimization problem can be formulated as follows:

$$
\min _{\eta} f(\eta)
$$

the constraints are:

$$
\begin{aligned}
g_{j}(x) \leq \overline{g_{j}}, \quad j & =[1,2, \ldots, K], \\
\frac{1}{V_{0}} \sum_{i=1}^{N} \eta_{i} & \leq F,
\end{aligned}
$$

\footnotetext{
*e-mail: mrzyglod@mech.pk.edu.pl
}

where $x=\left[x_{1}, x_{2}, \ldots, x_{N}\right]$ is a vector of finite elements; $\eta=\left[\eta_{1}, \eta_{2}, \ldots, \eta_{N}\right]$ is a vector of design variables defined as $\eta_{i}=E_{i} / E_{0}, E_{i}$ and $E_{0}$ are respectively, intermediate and real material Young's modules; $g_{j}(x)$ are the criterion parameters; $\overline{g_{j}}$ are the upper bounds of constraints; $V_{o}$ is the starting point of volume of the structure limited by design space $\Omega ; f(\eta)$ is the objective function (the volume of the structure); $F$ is a volume fraction. The $N$ design variables represent a pseudo-density (stiffness parameter) of each finite element of the structures that vary between $\eta_{\min }$ and 1 . The lower boundary of pseudo-density $\eta_{\min }$ is introduced to prevent singularity of the equilibrium problem.

The constant criterion surface algorithm is a "hard-kill" type method, in which the topology of the structure is generated by iterative elimination of elements of a low value of a criterion function $g$. This method is similar to the ESO approach introduced by Xie and Steven [2].

In the CCSA algorithm, the removal procedure is controlled by a $\Delta F$ parameter of volume percentage reduction, which gives a possibility to control the optimization 'speed' (see Fig. 1). To define the $\Delta F$, a constraint criterion increasing parameter $\Delta g$ is calculated at every iteration. In result of such action the surface of constant constraint criterion is obtained in the optimized structure. The idea of shaping structures in the form of the surface of constant stresses was first proposed by Mattheck and Burkhardt [11].

Mattheck's idea is based on the mechanism of tree growth which always takes the shape of constant surface stress. In the method the structure increases its volume according to the law:

$$
\dot{\varepsilon}_{V}=k\left(\sigma_{v m}-\sigma_{r e f}\right)
$$

where $\dot{\varepsilon}_{V}$ - volume growth, $\sigma_{v m}-$ is the von Mises stress, $\sigma_{\text {ref }}$ is a reference stress. 


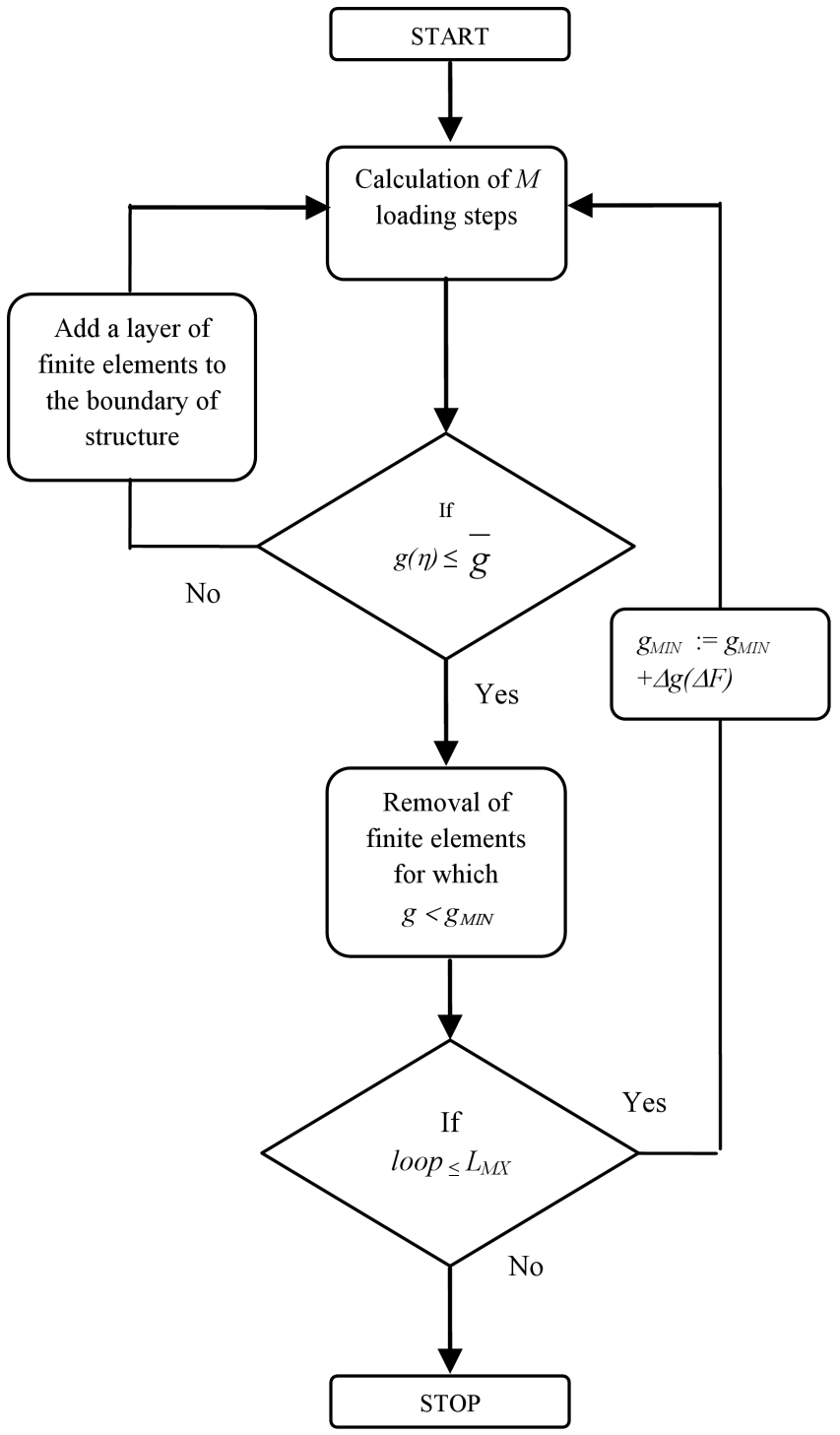

Fig. 1. The constant criterion surface algorithm

Mattheck suggested that inserting the squares of the stresses into Eq. (4) would result in homogenous distribution of elastic energies at the surface.

The condition of constant energy density at the free surface of the optimized structure was first deduceed by Wasiutyński [12]. However, the sufficient conditions were set in the work of Mróz [13]. Considerations of this issue can also be found in the work of Brandt [14] and Dems and Mróz [15].

The stress-constrained topology optimization procedure can give premature results when it is stuck in point of high values of the state parameter. The original ESO method allowed only for the elimination of the elements satisfying the following condition:

$$
\frac{\sigma_{e}^{v m}}{\sigma_{\max }^{v m}}<R R_{i}
$$

where $\sigma_{e}^{v m}$ is equivalent von Mises stress, $\sigma_{\max }^{v m}$ is maximum admissible von Mises stress, $R R_{i}$ is rejection rate parameter.
However, Querin et al. proposed a modified version called BESO (Bi-directional Evolutionary Structural Optimization) which allowed also to add new elements [16]. In the BESO method, new elements are added in the vicinity of the existing elements with high values of the criterion function.

In the CCSA the element removing procedure also starts from the selection of elements with the smallest value of criterion function. However, the boundary level of parameter criterion is chosen for each iteration in order to obtain the constant value of the removed material volume defined by the parameter $\Delta F$.

In comparison with the BESO, CCSA has a different method of increasing the volume of the structure called 'layer expansion algorithm' [17]. In this procedure when criterion function is over the limit, a layer of finite elements is added to the entire boundary of the structure (see Fig. 2). The process of volume expansion is continued until the criterion parameter $g$ returns to admissible values. As it was observed, by increasing and decreasing the structure volume, the algorithm obtains better solutions after every breakdown on the local quasi optimum. This scheme is analogous to the simulated annealing (SA) [18]. In this method the new solution depends on a global parameter $\mathrm{T}$ (called the temperature), that is gradually decreased during the process. The SA algorithm allows also to increase parameter $\mathrm{T}$, what potentially prevents stocking at local optima.
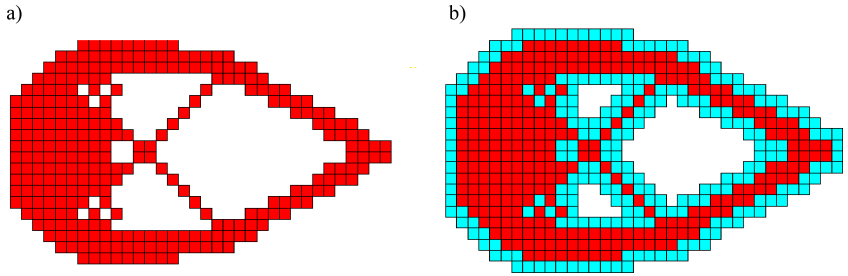

Fig. 2. The layer expansion algorithm: a structure before (a) and after operation of adding a layer of finite elements to the structure boundary (b)

It should be noted that there is similarity of CSSA to two other optimization methods in which there are local rules to modify the structure. The first is the bio-inspired remodeling method introduced by Nowak [19]. The remodeling algorithm is based on trabecular bone surface adaptation phenomenon. In the remodeling procedure the process of material adding or removing is controlled by a special range of Strain Energy Density called 'Lazy zone'. The remodeling algorithm compared to CCSA is a more complex procedure.

Cellular Automata (CA) as described in the work of Tovar et al. [20], Bochenek and Tajs-Zielinska [21], is the second of the optimization methods in which some similarity to the CCSA algorithm can be found. The main and fundamental difference between CA and the CCSA is in the local nature of the CA procedure and its 'one-way' model optimization. The $\mathrm{CA}$ procedure starts from a minimum volume of the structure and increases the volume according to the local rules of the upgrade. In the same way it is possible that the algo- 


\section{Multi-constrained topology optimization using constant criterion surface algorithm}

rithm CCSA begins work from a minimum volume but has a steady increase in volume until the global constraints (such as allowable stress) are met.

In the compliance constrained optimization for the structural optimization problems with multi-loads, the weighted sum of the compliance is usually used. The CCSA method to take into account forces that act in the asynchronous way on the structure, a 'compare and save maximum' procedure of summation of constrain criterion values is proposed [22]. The procedure assumes, that during each iteration for every finite element only the maximum values of the constraint criterion of all load cases will be written to the equivalent vector. This procedure is based on the 'Rainflow Cycle Counting' method used in the fatigue analysis [23].

To test the convergence of the algorithm, several benchmark problems were used with application of stress constraint [24-27]. In Figs. 3a-d and 4a-c the results of the tests are presented. The obtained numerical solutions are consistent with the data published in the literature for the compliance and stress constraints [28-31]. However, the numerical solution of Sokół-Lewiński problem (4c) [27] is probably published for the first time.

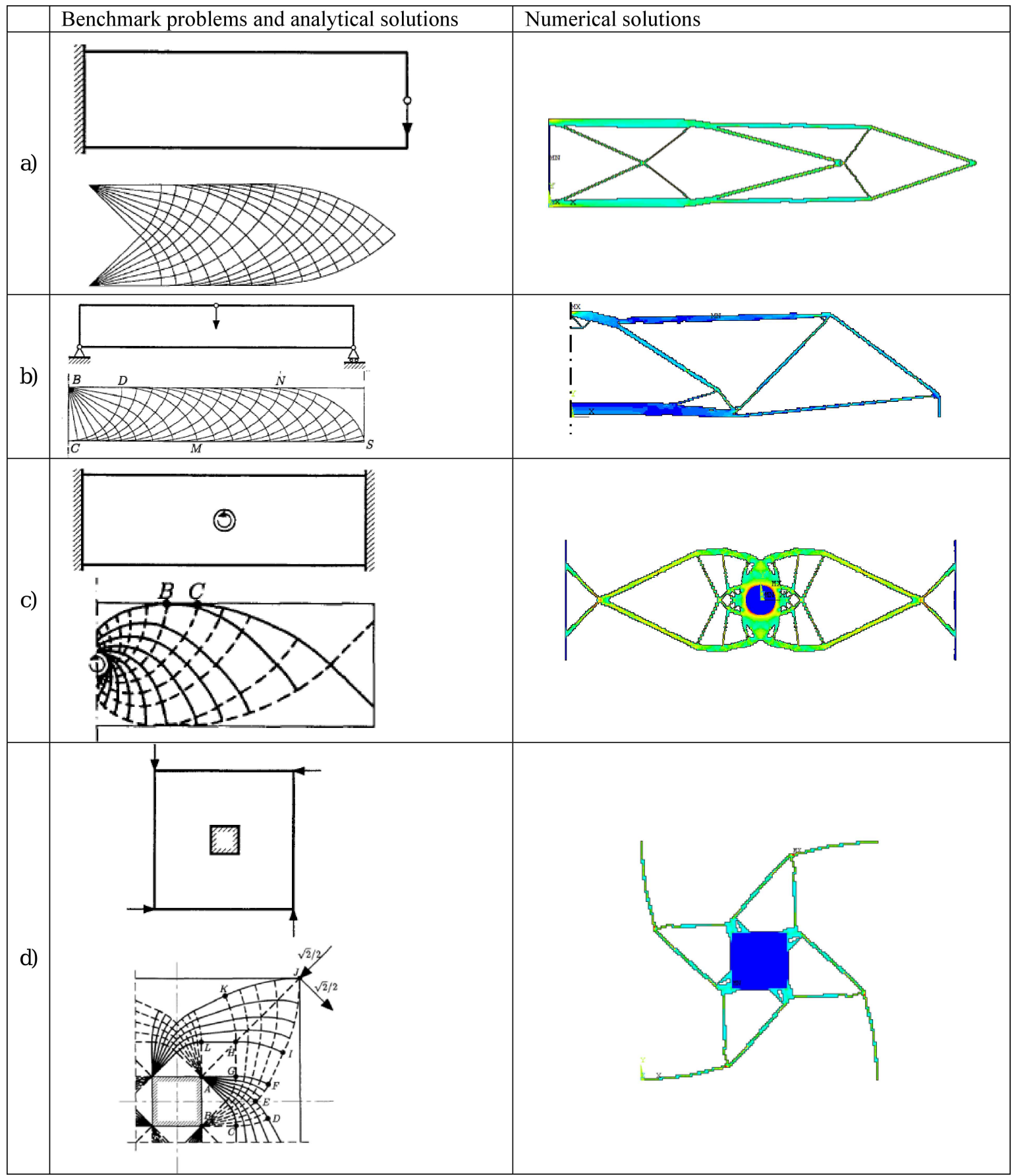

Fig. 3. Results of benchmark problem tests part I: after Ref. [24] 
M. Mrzygłód

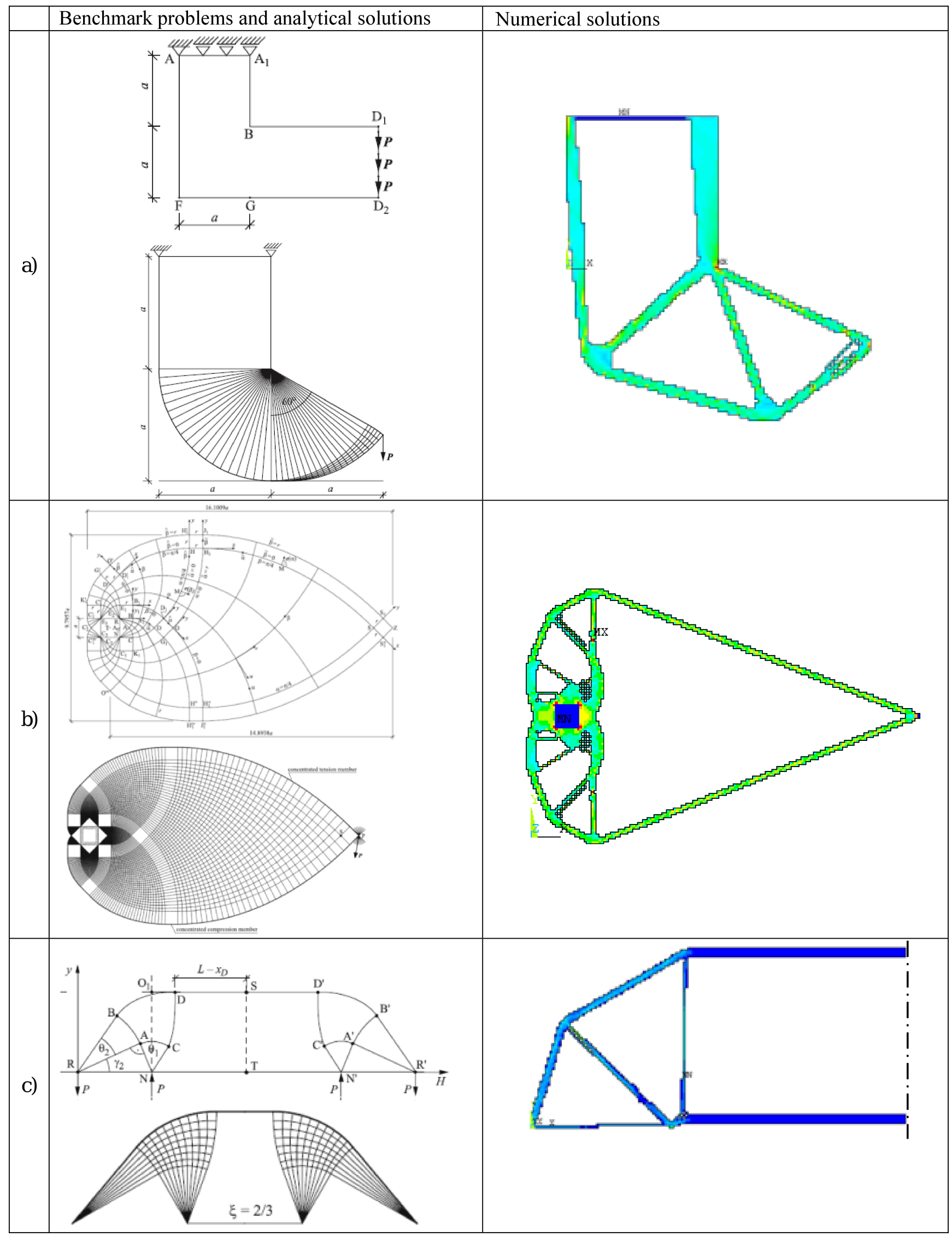

Fig. 4. Results of benchmark problem tests part II after Refs. [25-27] 
Multi-constrained topology optimization using constant criterion surface algorithm

\section{Multi-constrained topology optimization}

For multi-constrained topology optimization problems following normalized constraints are introduced:

$$
g_{n j}(x) \leq \frac{g_{j}(x)}{\overline{g_{j}}} \leq 1, \quad j=[1,2, \ldots, K],
$$

where $g_{n j}(x)$ are the normalized criterion parameters.

To consider an influence of different constraints on the optimized structure, a new constraint summation method is applied. For every iteration an equivalent design space $\Omega_{e q}$ is constructed, which is only the mathematical representation of the design space $\Omega$ The equivalent design space for all normalized constraints is calculated according to "compare and save max' rules. It means that only maximum values among compared $g_{n j}$ vectors are transferred to the resulting equivalent design space. The normalized constraints of final equivalent design space are used by the constant criterion surface algorithm of topology optimization.

\section{Examples of multi-constrained optimization}

Example 1. The problem of L-shaped domain optimization with horizontal line support and complex loads was selected as an optimization example. The example is based on the Lewiński-Rozvany analytical benchmarks for topological optimization III [25] (Fig. 5a). The FE model of the example structure with boundary conditions is shown in Fig. 5b, $N=7500$. A pulsating $(R=0)$ load was applied for the chosen structure. For a multi-constrained test, two types of constraints were used, the Dang Van high-cycle fatigue and von Mises equivalent stress criteria $(K=2)$. a)

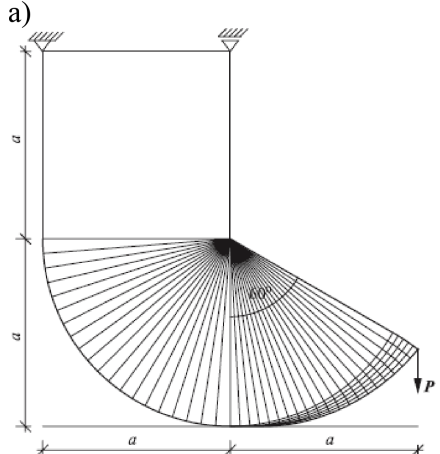

b)

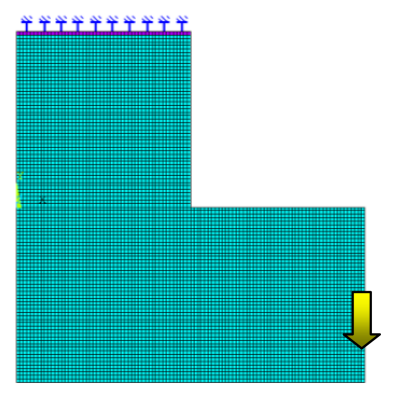

Fig. 5. Analytical solution [25] (a) and FE model (b) with displacement boundary conditions and pulsating load $(R=0)$

The Dang Van criterion is as follows [5, 32]:

$$
\max _{A}\left[\tau(t)+\kappa \sigma_{H}(t)\right] \leq \lambda,
$$

where $A$ is the area of studied object,

$$
\begin{gathered}
\tau(t)=\frac{\sigma_{1}(t)-\sigma_{3}(t)}{2}, \\
\sigma_{H}(t)=\frac{1}{3}\left(\sigma_{1}(t)+\sigma_{2}(t)+\sigma_{3}(t)\right), \\
\lambda=t_{-1},
\end{gathered}
$$

$$
\kappa=3 t_{-1} / f_{-1}-3 / 2
$$

$\left(f_{-1}, t_{-1}\right.$ stand for reversed bending and reversed torsion fatigue limits).

For an assumed material model $\left(f_{-1}=190 \mathrm{MPa}, t_{-1}=\right.$ $114 \mathrm{MPa}, \sigma_{f}=205 \mathrm{MPa}$ ) the topology optimization results for a single constraint (von Mises or Dang Van) are presented in Figs. 6 and 7.

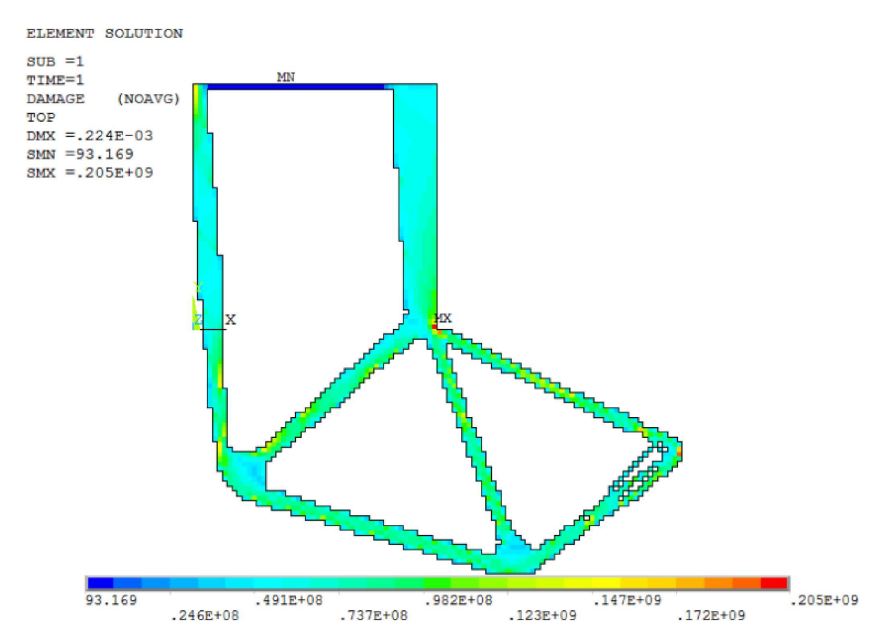

Fig. 6. The topology optimization results for von Mises constraint $\overline{g_{1}}=205 \mathrm{MPa} ; F=21.2 \%$

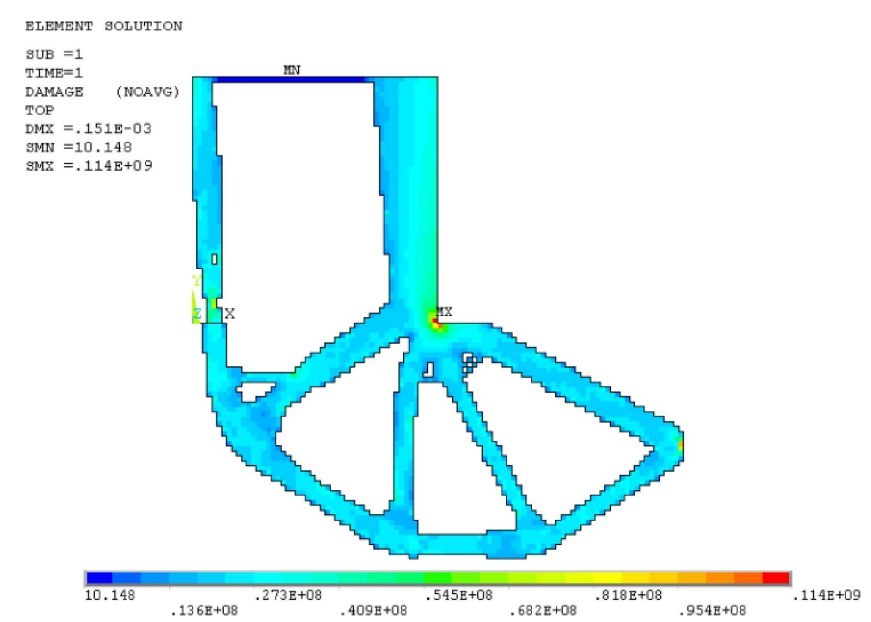

Fig. 7. The topology optimization result for Dang Van constraint $\overline{g_{2}}=114 \mathrm{MPa} ; F=32.2 \%$

The topology optimization result for multi-constraints (Dang Van and von Mises) is presented in Fig. 8.

The application of the fatigue or fatigue-stress constraints gave similar results (see Figs. 7 and 8). Whereas, the stress constraint allowed for the biggest volume reduction. From the above examples it can be concluded that the addition of the fatigue constraint has an important influence on the optimum structure layout. The differences in volume between examples with fatigue constraints show the accuracy of the algorithm that depends on the $\Delta F$ parameter $(\sim 1 \%)$ (see Fig. 1). 


\section{Mrzygłód}

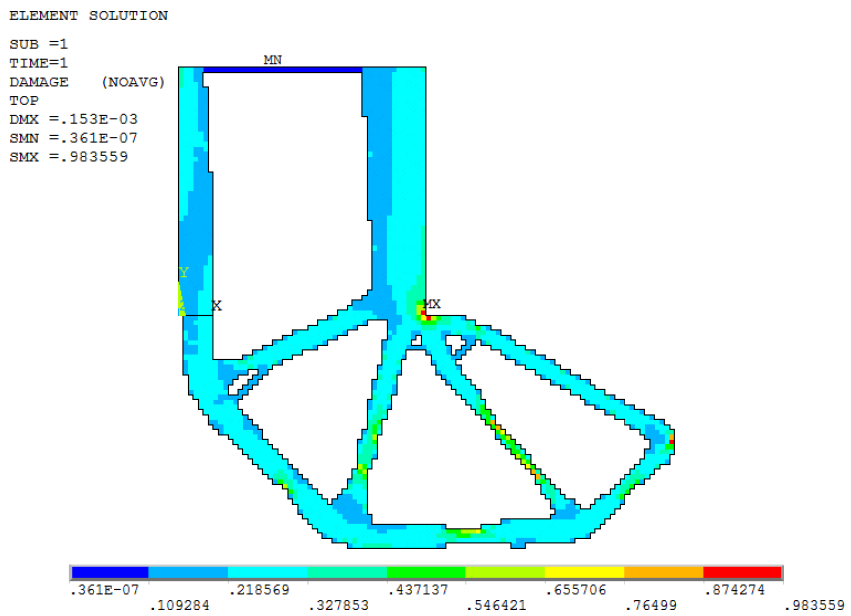

Fig. 8. The topology optimization result for multi-constraint (Dang Van and von Mises), $\overline{g_{n}}=1 ; F=30.8 \%$

Example 2. In the second example, the influence fatigue constraint on the L-shaped structure was widely examined.
The fatigue load $\left(F_{f}\right)$ was reduced to $100 \mathrm{~N}$ and various directions of this load was considered. The results of topology optimization for static $\left(F_{s}\right)$ and fatigue loads are shown in Fig. 8a-c.

As we see from results of example II, the small value of fatigue load does not make a significant impact on the structure volume. However, it has clear influence on the layout of the structure (see Fig. 9a-c).

Example 3. For the second load case of example II (Fig. 7b: $F_{s}=-250 \mathrm{~N}(y) ; F_{f}=-100 \mathrm{~N}(x)$ ), a compliance value (strain energy) was calculated (see Fig. 10). The compliance value has been used to the single constrained optimization (see Fig. 11). Finally, the multi-constrained optimization was conducted for stress, fatigue and compliance constraints $(K=3)$. From the result of optimization shown in Fig. 12, we can find that the compliance constraint has an influence on the volume of the structure. The single compliance constraint formed a complex layout of structure.

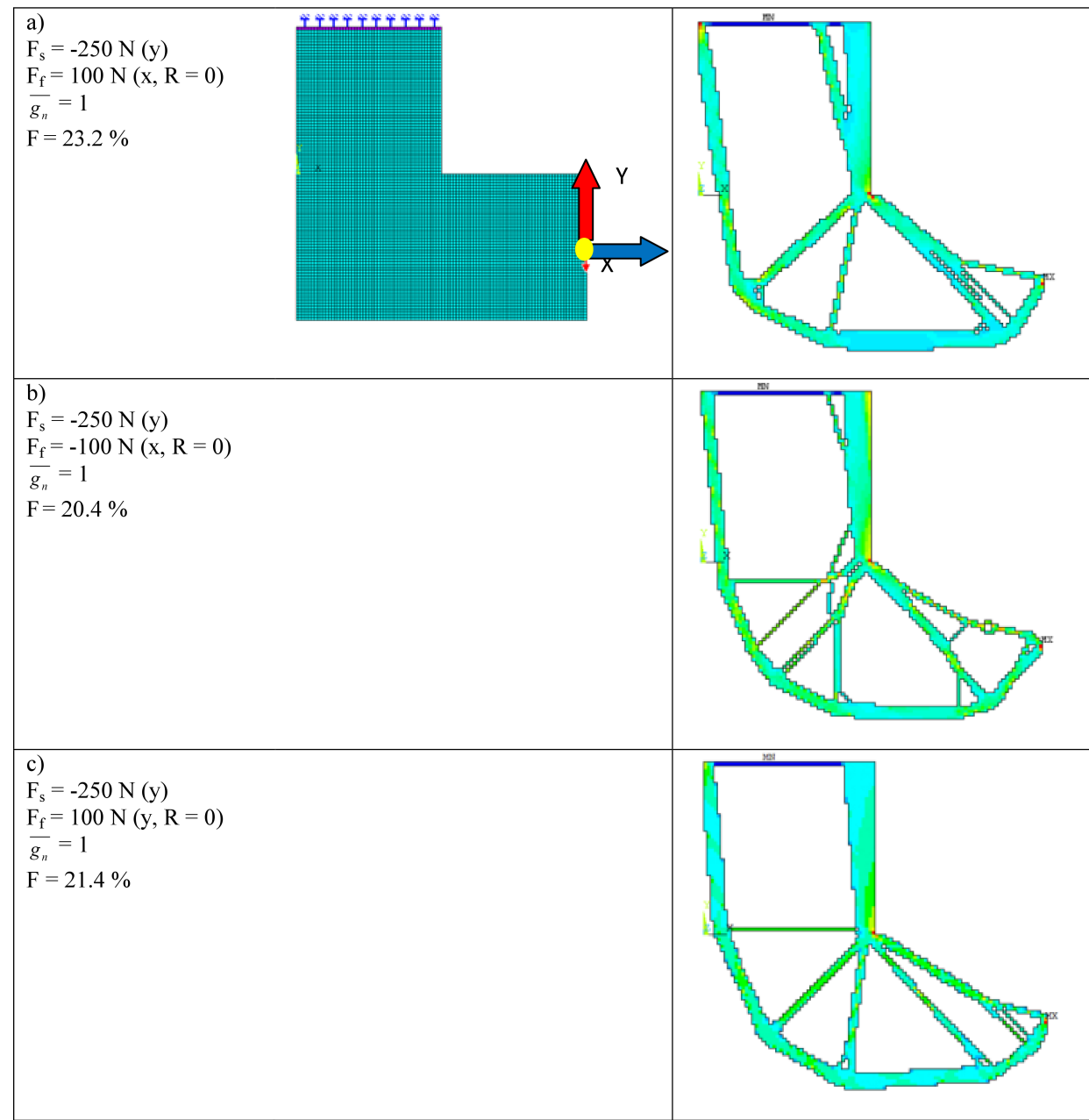

Fig. 9. The topology optimization results for the second example; $F_{s}-$ is static load force; $F_{f}-$ is fatigue load force 
Multi-constrained topology optimization using constant criterion surface algorithm

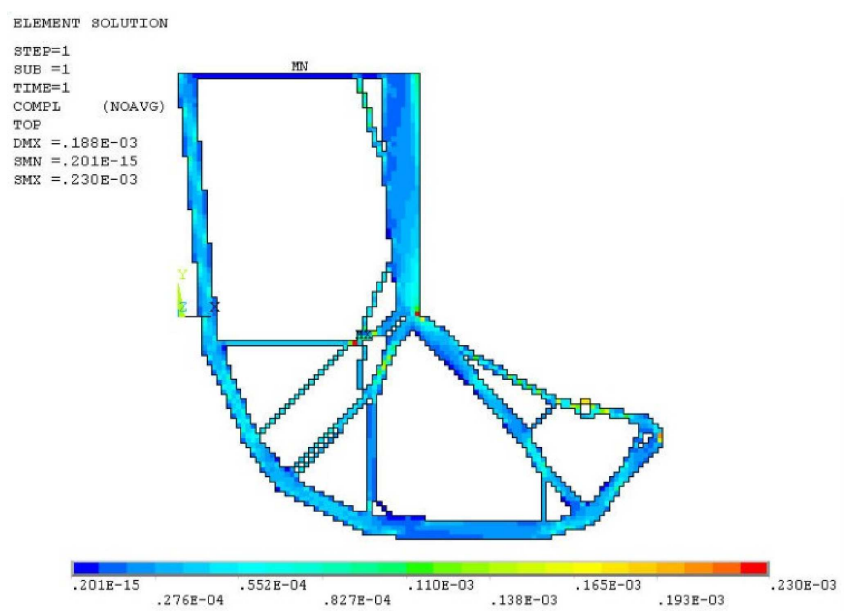

Fig. 10. Result of the compliance value test for the second load case of example 2 (see Fig. 7b), contour map of the $g_{j}(x)$ criterion function
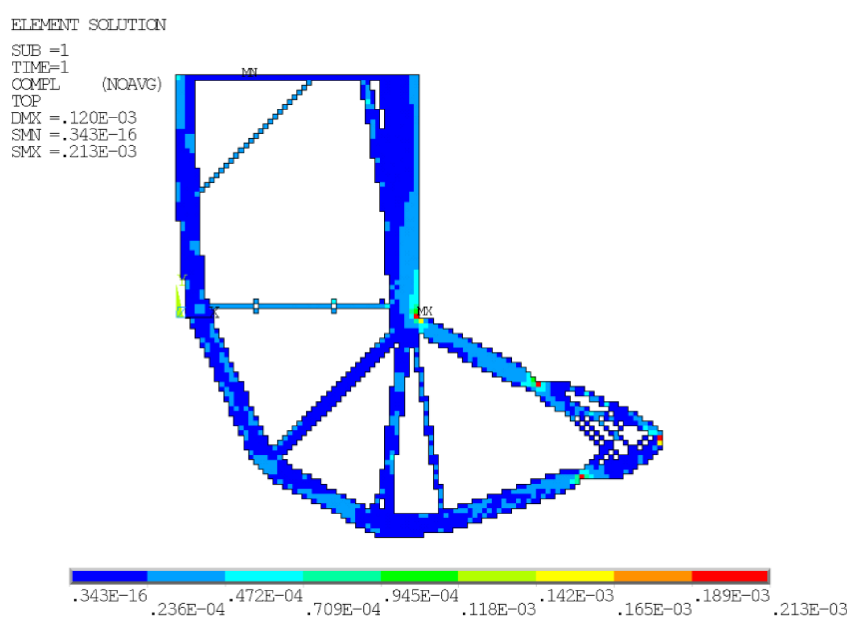

Fig. 11. The topology optimization result of the compliance constraint, $\overline{g_{3}}=0.23 \mathrm{e}-3 ; F=23.4 \%$

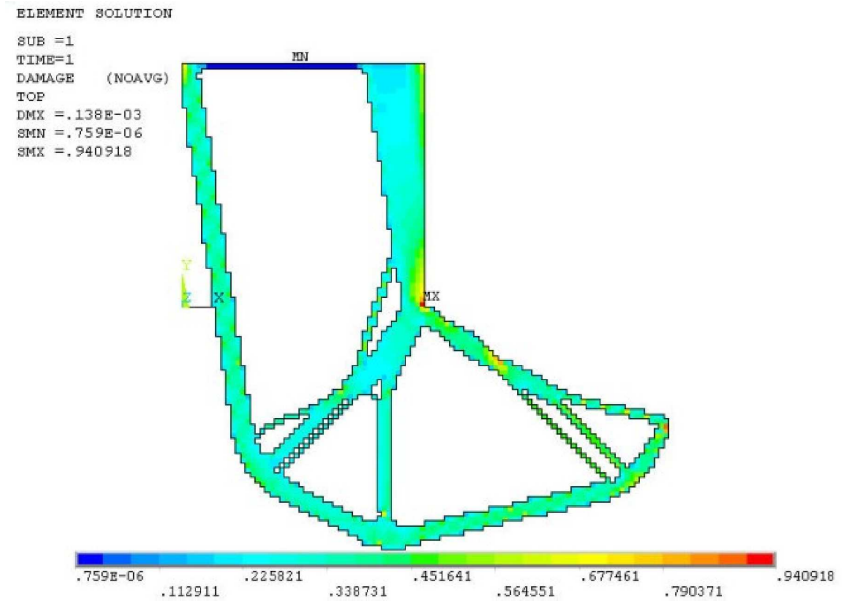

Fig. 12. The topology optimization result for stress (von Mises), fatigue (Dang Van) and compliance constraints, $\overline{g_{n}}=1 ; F=23.3 \%$

\section{Conclusions}

In this article, the constant criterion surface algorithm with multi-constraints was presented. As illustrated in the examples, the application of normalized constraints and equivalent design space to topology optimization gives a possibility to solve the optimization problem with stress, fatigue and compliance constraints. In the optimization investigation, different layouts were obtained for single and multi-constrained optimization. The results of examples 13 , showed a significant difference between the fatigue and von Mises equivalent stress constraints impact on the optimized structure layout. These results are compatible with the work of Mrzyglod [33]. However, the results of optimization with multi-constraints of the L-Bracket example have not had yet comparable references in the literature. Moreover, the concept of normalized constraints in conjunction with the equivalent design space is also presented for the first time.

The presented methodology addresses the need of finding an easy in application method of optimization with complex constraints, which has the particular meaning for the industrial application. It seems that the method of constraints summation, proposed in the article, can also be transferred to the commonly used methods like SIMP or ESO/BESO $[1,2,16,29]$, and replace currently in use the procedure of the weighted sum of normed constraints.

\section{REFERENCES}

[1] M. Zhou and G.I.N. Rozvany, "The COC algorithm. Part II: topological, geometrical and generalized shape optimization", Comp. Meth. Appl. Eng. 89, 309-336 (1991).

[2] Y.M. Xie and G.P. Steven, "A simple evolutionary procedure for structural optimization", Comput. Struct. 49, 885-896 (1993).

[3] M. Bendsoe and O. Sigmund, Topology Optimization. Theory, Methods, and Applications, Springer, New York, 2003.

[4] B. Desmorat and R. Desmorat, "Topology optimization in damage governed low cycle fatigue", Mecanique 336, 448-453 (2008).

[5] M. Mrzyglod, "Multiaxial HCF and LCF constraints in topology optimization”, Proc 9-th Int Conf on Multiaxial Fatigue \& Fracture 1, 803-810 (2010).

[6] P. Honarmandi, J.W. Zu, and K. Behdinan, "Reliability-based design optimization of cantilever beams under fatigue constraint", AIAA J. 45 (11), 2737-2746 (2007).

[7] M. Bruggi and P. Venini, "A mixed FEM approach to stressconstrained topology optimization", Int. J. Num. Meth. Eng. 73 (12), 1693-1714 (2008)

[8] S. Min S. Nishiwaki, and N. Kikuchi, "Unified topology design of static and vibrating structures using multiobjective optimization", Computers and Structures 75, 93-116 (2000).

[9] D.-C. Lee, HS. Choi, and C.S. Han, "Design of automotive body structure using multicriteria optimization", Struc. Multidisc. Optim. 32 (2), 161-167 (2006).

[10] A. Ramani, "Multi-material topology optimization with strength constraints", Struc. Multidisc. Optim. 43, 597-615 (2011). 
[11] C. Mattheck and S. Burkhardt, "A new method of structural shape optimisation based on biological growth", Int. J. Fatigue 12 (3), 185-190 (1990)

[12] Z. Wasiutyński, "On the congruency of the forming according to the minimum potential energy with that according to equal strength", Bull. Pol. Ac.: Tech. 8 (6), 259-268 (1960)

[13] Z. Mróz, "On a problem of minimum weight design", $Q$. Appl. Math. 19, 127-135 (1961).

[14] A.M. Brandt, Criteria and Methods of Structural Optimization, PWN, Warszawa, 1984.

[15] K. Dems and Z. Mróz, "Multiparameter structural shape optimization by finite element method", Int. J. Num. Meth. Eng. 13, 247-263 (1978).

[16] O.M. Querin, G.P. Steven, and Y.M. Xie, "Evolutionary structural optimization (ESO) using bidirectional algorithm", Eng. Comp. 15, 1031-104 (1998).

[17] M. Mrzyglod, "Using layer expansion algorithm in topology optimization with stress constraints", Proc. CMM-2009 - Computer Methods in Mechanics 1, 319-320 (2009).

[18] S. Kirkpatrick, C.D. Gelatt, and M.P. Vecchi, "Optimization by simulated annealing", Science 220, 671-680 (1983).

[19] M. Nowak, "Structural optimization system based on trabecular bone surface adaptation", Struc. Multidisc. Optim. 32 (3), 241-249 (2006).

[20] A. Tovar, N.M. Patel, G.L. Niebur, M. Sen, and J.E. Renaud, "Topology optimization using a hybrid cellular automaton method with local control rules", ASME J. Mechanical Design 128 (6), 1205-1216 (2006)

[21] B. Bochenek and K. Tajs-Zielińska, "Novel local rules of cellular automata applied to topology and size optimization", Eng. Optimization 44 (1), 23-25 (2012).

[22] M. Mrzyglod, "Two-stage optimization method with fatigue constraints for thin-walled structures", J. Theoretical and Applied Mechanics 48 (3), 567-578 (2010).

[23] M. Matsuishi and T. Endo, "Fatigue of metals subjected to varying stress-fatigue lives under random loading", Proc. Kyushu District Meeting, JSEM 1, 37-40 (1968).

[24] G.I.N. Rozvany "Exact analytical solutions for some popular benchmark problems in topology optimization", Struct. Optim. 15, 42-48 (1998).

[25] T. Lewiński and G.I.N. Rozvany, "Exact analytical solutions for some popular benchmark problems in topology optimization III: L-shaped domains", Struct. Multidisc. Optim. 35, 165-174 (2008).

[26] T. Lewiński and G.I.N. Rozvany, "Analytical benchmarks for topology optimization IV: square-shaped line support", Struct. Multidisc. Optim. 36, 143-158 (2008).

[27] T. Sokół and T. Lewiński, "On the solution of the three forces problem and its application to optimal designing of a certain class of symmetric plane frameworks of least weight", Struct. Multidisc. Optim. 42, 835-853 (2010).

[28] H.A. Eschenauer and N. Olhoff, "Topology optimization of continuum structures: a review", Applied Mechanics Reviews 54 (4), 331-389 (2001).

[29] M. Bendsoe and O. Sigmund, Topology Optimization. Theory, Methods, and Applications, Springer, New York, 2003.

[30] G. Chiandussi, "On the solution of a minimum compliance topology optimisation problem by optimality criteria without a priori volume constraint specification", Comput. Mech. 38, 77-99 (2006).

[31] A. Ramani, "Multi-material topology optimization with strength constraints", Struct. Multidisc. Optim. 43, 597-615 (2011).

[32] K. Dang Van, B. Griveau, and O. Message, "On a new multiaxial fatigue limit criterion: theory and application", in Biaxial and Multiaxial Fatigue, pp. 479-496, Mechanical Engineering Publications, London, 1989.

[33] M. Mrzyglod, "Multiaxial HCF and LCF constraints in topology optimization", Proc. 9-th Int. Conf. on Multiaxial Fatigue \& Fracture, ICMFF9 1, 803-809 (2010). 